\title{
BMJ Open Impact of National Drug Pricing Policy 2018 on access to medicines in Lahore division, Pakistan: a pre-post survey study using WHO/HAI methodology
}

To cite: Saeed A, Saeed H, Saleem Z, et al. Impact of National Drug Pricing Policy 2018 on access to medicines in Lahore division, Pakistan: a prepost survey study using WHO/ HAl methodology. BMJ Open 2020;10:e034720. doi:10.1136/ bmjopen-2019-034720

- Prepublication history and additional material for this paper are available online. To view these files, please visit the journal online (http://dx.doi. org/10.1136/bmjopen-2019034720).

Received 03 October 2019 Revised 21 July 2020

Accepted 04 August 2020

Check for updates

(c) Author(s) (or their employer(s)) 2020. Re-use permitted under CC BY-NC. No commercial re-use. See rights and permissions. Published by BMJ.

For numbered affiliations see end of article.

Correspondence to

Dr Yu Fang;

yufang@mail.xjtu.edu.cn

\section{ABSTRACT}

Objective To evaluate the impact of new National Drug Pricing Policy (NDPP) 2018 on access to medicines in terms of prices, availability and affordability.

Design Two cross-sectional surveys were undertaken before and after the launch of NDPP 2018, using a modified WHO/Health Action International (WHO/HAl) methodology.

Setting Four districts of Lahore division, Pakistan. Participants 16 public sector hospitals and 16 private sector retail pharmacies.

Measures The pre and post survey data on prices and availability of lowest price generics (LPGs) and originator brands (OBs) of 50 medicines were obtained by visiting the same public and private sector health facilities $(n=32)$. Out of 50, 46 surveyed medicines were from the National Essential Medicines List. Inflation-adjusted median unit prices (MUPs) and median price ratios (MPRs) from 2019 were used for price comparison. Affordability was calculated in terms of number of days' wages required to get a standard treatment by the lowest paid unskilled government worker.

Results The overall mean percent availabilities remained poor in both years, that is, far less than $80 \%$. In the public sector, the mean percent availability of OBs improved from $6.8 \%$ to $33.1 \%$, whereas, in the case of LPGs, it was reduced from $35.1 \%$ to $9 \%$. In the private sector, the mean percent availability of both OBs and LPGs demonstrated slight improvements in 2019, that is, $55.0 \%-58.3 \%$ and $20.3 \%-32.3 \%$. The adjusted MUPs and MPRs of OBs significantly increased by a median of $4.29 \%$ (Wilcoxon test $p=0.001, p=0.0001$ ), whereas the adjusted MUPs and MPRs of LPGs increased by a median of $15.7 \%$ ( $p=0.002, p=0.0002)$. Overall, the affordability of many medicines for common ailments was reduced significantly in 2019.

Conclusions The availability of medicines slightly improved, except in the case of LPGs, which was reduced in the public sector. The implementation of NDPP 2018 led to increase in drug prices, making the standard treatment for some of the most prevalent ailments unaffordable. So verily, the drug pricing policy must be reviewed to ensure access to essential medicines.
Strengths and limitations of this study

- This study is the first attempt to estimate the impact of a drug pricing policy on the prices of essential medicines in four districts of Lahore division, Pakistan, using a validated WHO/Health Action International (WHO/HAl) methodology.

- The data were collected from the same health facilities in both years to make the comparison of results reliable.

- This study provides an objective evidence to the policy makers, in terms of impact of National Drug Pricing Policy (NDPP) 2018 on access to medicines, for improving the current pricing policies.

- The study is limited to only one division of Pakistan, although the medicine prices are fixed centrally and are supposed to be the same across the country, affecting generalisability of the findings.

- The cross-sectional design of the study might not reflect the long-term impact of NDPP 2018-the average monthly, quarterly or yearly availability of medicines at individual outlets.

\section{INTRODUCTION}

Access to affordable and quality assured essential medicines (EMs) is considered as a key component of an effective healthcare system. It has also been pledged under Sustainable Development Goal (SDG) 3 by the United Nations that the equitable access to affordable EMs will be ensured as a basic human right. ${ }^{12}$ Pakistani government, like many other low-income and middle-income countries, has been grappling with the issue of high medicine prices and poor availability of medicines that compromises the accessibility of medicines. ${ }^{13-6}$ In Pakistan, the medicines are provided free of cost at public sector health facilities, while patient pays out of pocket to get medicines from private sector. ${ }^{5}$ In fact, the poor availability of medicines at 
public sector compels the patients to buy medicines from the private sector that escalates the burden on patient's pocket, as 24.3\% (in 2015) of the population is living below the national poverty line. ${ }^{3478}$ Besides, medicine prices have increased up to $100 \%$, both legally or illegally, in the past few years. ${ }^{3}$ The drug prices are fixed by the federal government and the National Health Services Regulation and Coordination (NHSRC). The regional drug inspectors (DIs) are responsible for monitoring drug prices in the pharmacies of their area. The NHSRC has been taking different policy measures to curb these issues through Drug Regulatory Authority of Pakistan (DRAP). The first ever National Drug Pricing Policy (NDPP) was launched in 2015 for making the pricing mechanism transparent but it had minimal impact on medicine prices, suitable for both patients and manufacturers as per media reports and available literature evidence. ${ }^{39}$ So, a new drug pricing policy was launched in 2018. ${ }^{10}$ The objectives of this policy were to improve access to EMs, devise rational prices, ensure a transparent mechanism for medicine pricing and discourage illegal increase in drug prices.

Many modifications have been made to the pricing strategies in the NDPP 2018 compared with NDPP 2015, importantly the inclusion of all drugs $(n=414)$ from National Essential Medicines List (NEML) under scheduled drugs category where the drugs are kept under strict price control as compared with other drugs. Whereas in NDPP 2015, only 160 drugs from NEML were enlisted in this category. ${ }^{9} 10$ In NDPP 2018, the annual adjustment in prices has been linked to the Consumer Price Index (CPI), the maximum retail prices (MRPs) of scheduled drugs (all drugs from NEML) could be increased up to $70 \%$ of the CPI, whereas the MRPs of all other drugs could be increased equivalent to CPI of the immediate preceding year. This step seems to improve the affordability of EMs for patients in Pakistan. If several generics are already available in the market, then in NDPP 2015, the MRP of new entrant was fixed by taking the average of other generics, while in NDPP 2018, MRP will be fixed equivalent to the highest MRP of the available generics. ${ }^{910}$ However, this would lead to even higher priced generics in the market that could compromise patient's affordability. Some media reports are claiming that the current increase (up to 200\%) in medicine prices is the highest in the last 40 years, while others are claiming that government is taking action against this illegal rise in medicine prices. ${ }^{11} 12$ However, there is no objective evidence to prove or disapprove these claims. The NDPP 2018 allows the MRPs of the new chemical entities (NCEs) to be fixed by using the external reference pricing (ERP) mechanism by considering India, Bangladesh, Indonesia, Sri Lanka, Philippines, Lebanon and Malaysia as reference countries. However, the reason behind using the MRPs of these countries as reference is not clear, although some of them practise free market economy model and do not impose any price control measures over the MRPs in community pharmacies that may lead to high prices. The NDPP 2018 also takes into account the wholesale or procurement prices from British National Formulary, Australian Pharmaceutical Benefit Scheme and New Zealand Pharmaceutical Management Agency while fixing the MRPs of NCEs; however, these may not be the true prices because discounts and rebates are given as a common practice, while making the payments. So, these ambiguities in the policy necessitate the evaluation of the actual impact of these policies on access to medicines in Pakistan.

In this context, we designed a study to measure the impact of new NDPP 2018 on access to EMs in terms of their prices, availability and affordability in Lahore division, Punjab. We undertook a survey after the implementation of NDPP 2018 and compared it with a similar survey performed before the launch of this policy in 2017. Considering the objectives of the NDPP 2018, we hypothesised that it will improve the availability and affordability, meanwhile decrease the prices of EMs.

\section{METHODOLOGY \\ Study design}

Two cross-sectional studies were conducted in 2016-2017 and 2019 using a variant of WHO/Health Action International (WHO/HAI) methodology in four districts of Lahore division, Pakistan. ${ }^{13}$ Since the focus of this study was to measure the impact of NDPP 2018 in terms of changes in medicines prices, availability and affordability after its implementation, therefore, the data on these parameters were collected to evaluate the accessibility of medicines in both years. ${ }^{10}$ The first survey was conducted from November 2016 to March 2017, while the second survey was from March to May 2019, representing two fiscal years of Pakistan. For optimal and reliable comparison, the lists of medicines, survey region and survey outlets selected for the survey 2019 were similar to those selected for 2017. The details of survey region, medicine selection, sampling of medicine outlets and data collection are given elsewhere and are briefly described in this paper. $^{7}$

\section{Survey areas}

Pakistan consists of four provinces that are subdivided into several administrative units called 'divisions', each division is further subdivided into districts, and districts into tehsils. Lahore is the largest division of Pakistan in terms of population, that is, 16.28 million (2017) and estimated to be 19.4 million as of 2018. ${ }^{14}$ It consists of four districts named Lahore, Kasur, Sheikhupura and Nankana Sahib, and 17 tehsils. All the four districts were selected for the surveys.

\section{Sampling of medicine outlets}

Medicine outlets or health facilities from both public and private sectors were sampled systematically using the WHO/HAI manual as a guiding principle in both the surveys. ${ }^{13}$ A total of 32 medicine outlets were surveyed (16 from the public sector and 16 from the private sector). 
From the public sector, hospitals from all three tiers of healthcare system, that is, primary, secondary and tertiary, were selected. One main hospital in each district was selected as a survey anchor along with additional three more hospitals selected randomly and situated within 3 hours' drive from the main hospital. In this way, four hospitals were selected in each district, making up a total of 16 hospitals from Lahore division. From the private sector, one registered pharmacy was selected, situated within $10 \mathrm{~km}$ range of each public sector hospital. So, a total of 16 pharmacies were selected from Lahore division, that is, 4 retail pharmacies from each district. It is important to note that each survey unit, one hospital and one nearby pharmacy, was located in different tehsils, so out of 17 tehsils of Lahore division, 16 were surveyed in both years.

\section{Selection of medicines}

Fifty medicines were selected for survey as per WHO/HAI methodology, which included all 14 medicines from the WHO core global list of medicines and 36 supplementary medicines. The criterion of selecting medicines for supplementary list was local disease burden and inclusion of medicines in NEML. ${ }^{15}$

\section{Data collection}

The data were collected using a data collection form by the trained data collectors. The data collectors visited the health facilities and physically checked the medicine prices and availability for both OBs and LPGs of each medicine and entered it into data collection forms. The data for each year were entered separately into the WHO/HAI workbook by using double data entry process, to avoid any mistake. ${ }^{13}$ The patient prices or the prices charged to patients were entered into the forms for private sector only, since the medicines are provided free of charge in the public sector, in Pakistan. Thus, the availability of medicines was documented only for public sector facility.

\section{Data analysis}

Data were analysed by using the WHO/HAI preprogrammed Excel workbook, ${ }^{13}$ IBM Statistical Package for the Social Sciences (SPSS) V.22.0 and R V.3.5.1 (codename 'Feather Spray').

\section{Availability}

Availability was calculated as percentage of particular medicine available at each facility on the day of data collection. The mean percentage availabilities were also calculated and compared between different sectors (public and private), product types (OBs and LPGs) and among different groups (global medicines, supplementary medicines, medicines from NEML, medicines used to treat non-communicable diseases (NCDs) and infectious diseases (IDs)). Availability was documented as follows: absent, $0 \%$ of facilities had surveyed enlisted medicines at the time of survey; low, $<50 \%$ of facilities had the surveyed enlisted medicines; fairly high, $50 \%-80 \%$ of facilities had the surveyed enlisted medicines; high, $>80 \%$ of facilities, survey enlisted medicines were found in most of the facilities. $^{1617}$

\section{Medicine prices}

Medicine prices were calculated as median unit prices (MUPs) in Pakistani rupees (PKR) and were also compared with international reference prices (IRPs) to calculate the median price ratios (MPRs). The IRPs were obtained from Management Sciences for Health (MSH) drug price indicator guide $2015 .{ }^{18}$ An MPR greater than 1 for the public sector and greater than 2 for the private sector would lodge any medicine into high priced medicines category. ${ }^{7}$ For comparing the prices between 2 years, the MUPs from 2019 were deflated by 3.33\%, taking 2017 as base year. Seventy per cent of the CPI was used for calculating this deflation factor because the medicine prices can be increased annually by $70 \%$ of the CPI as per NDPP $2018^{19}$, whereas MPR was calculated as follows:

Median price ratio $($ MPR $)=$ Median local unit price $/($ International reference unit price.

\section{Affordability}

Affordability for treatment of different common diseases with selected medicines was calculated and compared in terms of number of days' wages (NDWs) required for a lowest paid government employee to get the standard treatment courses. Whereas if a patient had to spend more than 1 day of his wage for treatment with a specific medicine in a month, that medicine was considered unaffordable. ${ }^{13}$ For affordability comparison, NDWs in 2 years, the prices in 2019 were not deflated because the salary has also been increased in 2019. So, the salary of the lowest paid unskilled government worker was taken as 14000PKR per month (2016-2017) and $15000 \mathrm{PKR}$ per month (2018-2019). ${ }^{20}$

\section{Comparative analysis}

Two patient prices were required to be included in the comparative analysis, one from 2017 and other from 2019. The difference in prices, availability and affordability was computed as percentage change for each product. The mean availability and MUPs were also compared between different categories of medicines (NEML and non-NEML medicines; global and supplementary medicines; medicines for IDs and NCDs) across the years. We compared affordability in terms of NDWs to get the standard treatment from the surveyed medicines. These were also compared among medicines for different disease groups (asthma, cardiovascular diseases (CVD), infectious diseases, brain disorders, diabetes, ulcer and arthritis; IDs and NCDs). To identify whether the difference between MUPs, MPRs and NDWs was significant across 2 years, we used Wilcoxon signed-rank test. We took $p<0.01$ as an indicator of significant difference in all the statistical testing. 


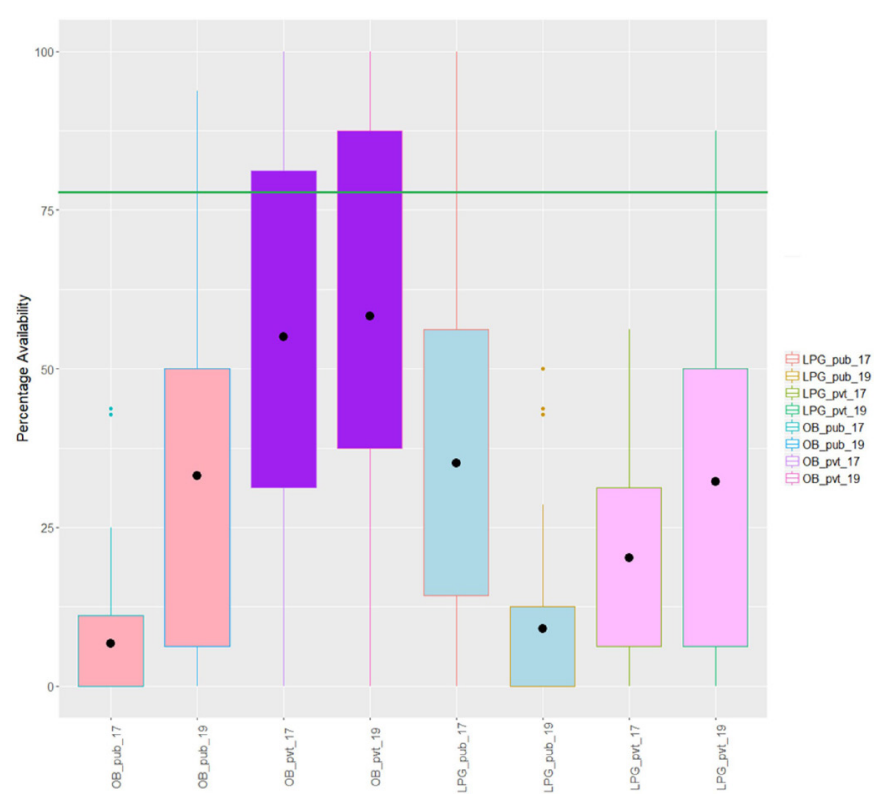

Figure 1 Box plot of percent availability of lowest price generics (LPGs) and originator brands (OBs) in both public (pub) and private (pvt) sectors in 2017 and 2019. This box plot shows the distributional characteristics of the percent availability of medicines in two groups (OB and LPG) for the years 2017 and 2019. The mean percent availability is represented by the dot inside the box.

\section{Patient and public involvement}

Patients or the public were not involved in the design, or conduct, or reporting or dissemination of this study.

\section{RESULTS}

\section{Availability of medicines}

The overall availability of surveyed medicines was improved in 2019 compared with 2017, except for LPGs in the public sector, where it demonstrated reduction.

Availability in the public sector

In the public sector, the availability of both OBs and LPGs was poor in both years. None of the mean availabilities touched the benchmark of $80 \%$. The mean percent availability of OBs improved from $6.8 \%$ to $33.1 \%$, whereas, in the case of LPGs, it reduced from $35.1 \%$ to $9 \%$. The individual percent availabilities of each medicine (OB and LPG) are given in online supplemental table S1. For better understanding of the data, we have used the data visualisation tool in $\mathrm{R}$ and plotted a box plot of the data, as shown in figure 1 . The box plot showed distributional characteristics of the percent availability of medicines in two groups (OB and LPG) for the years 2017 and 2019. The mean percent availability is represented by the dot inside the box. In 2017, availabilities of $75 \%$ of the OBs in the public sector were less than $11.2 \%$, as shown by the third quartile or the upper bar of the first box plot. Whereas, in 2019, $75 \%$ of the OBs had availabilities higher than $6.3 \%$, which is almost equivalent to the mean percent availability $(6.8 \%)$ in 2017 . This indicates a substantial increase in the availability of OBs in the public sector. In 2019, $75 \%$ of the LPGs had the availability of less than $12.5 \%$ (third quartile), whereas before the implementation of the NDPP 2018, $75 \%$ of the LPGs had availabilities of more than $14.3 \%$ (first quartile). This showed a remarkable decrease in the availability of LPGs in the public sector in 2019 .

\section{Availability in private sector}

In both years, the overall availability was better in the private sector than the public sector. Mean percent availability of both OBs and LPGs were improved in 2019, that is, $55.0 \%-58.3 \%$ and $20.3 \%-32.3 \%$, respectively. Availability of LPGs was less than OBs in both years. For OBs, the mean percent availability improved slightly by $3.3 \%$, while data distribution remained almost the same across the years, as shown in the box plot (figure 1). For LPGs, a substantial increase in the availability was observed in terms of mean percent availability, maximum value and change in data distribution (figure 1). In 2017, the IQR (range between upper and lower bar of the box) for percentage availability of LPGs ranged from $6 \%$ to $31 \%$, whereas it got improved in 2019 and ranged from $6 \%$ to $50 \%$.

\section{Availability in different subgroups of medicines}

When we compared availability of LPGs and OBs in different subgroups of medicines, as shown in table 1 , we found that mean percent availabilities of all global list medicines were higher than supplementary medicines in both years. Similarly, the availability of NEML medicines was higher than non-NEML medicines except for the non-NEML LPGs in the public sector, which was higher than NEML LPGs (19.2\% vs 8.1\%). In 2017, in the public sector, the availability of OBs for NCD medicines $(6.7 \%)$ was lower than OBs for ID medicines $(7.7 \%)$, whereas the availability of LPGs $(35.9 \%)$ was better than LPGs of ID medicines (33\%). Surprisingly, in 2019, the situation is inversed completely for NCD medicines, increased and decreased availability of OBs (33.2\%) and LPGs (33.1\%), respectively, whereas the availability decreased for LPGs $(8.8 \%)$ and increased for OBs $(9.5 \%)$ for ID medicines.

\section{Availability at different levels of healthcare}

When we compared the availability at different levels of public healthcare sectors, that is, primary, secondary and tertiary, the availability of OBs improved in 2019, while it decreased for LPGs. The pattern of overall medicine availability remained almost the same as of 2017, that is, tertiary care $>$ secondary care $>$ primarycare, as shown in online supplemental table S2.

\section{Medicine prices}

An overall increase was noted in all adjusted MUPs and adjusted MPRs between 2017 and 2019 for both OBs and LPGs, as shown in table 2. In 2019, for all 42 available OBs, the adjusted MUPs and MPRs significantly increased by a median of $4.3 \%$ (Wilcoxon test $\mathrm{p}=0.001$, $\mathrm{p}=0.0001$ ), whereas in the case of all 37 available LPGs, 


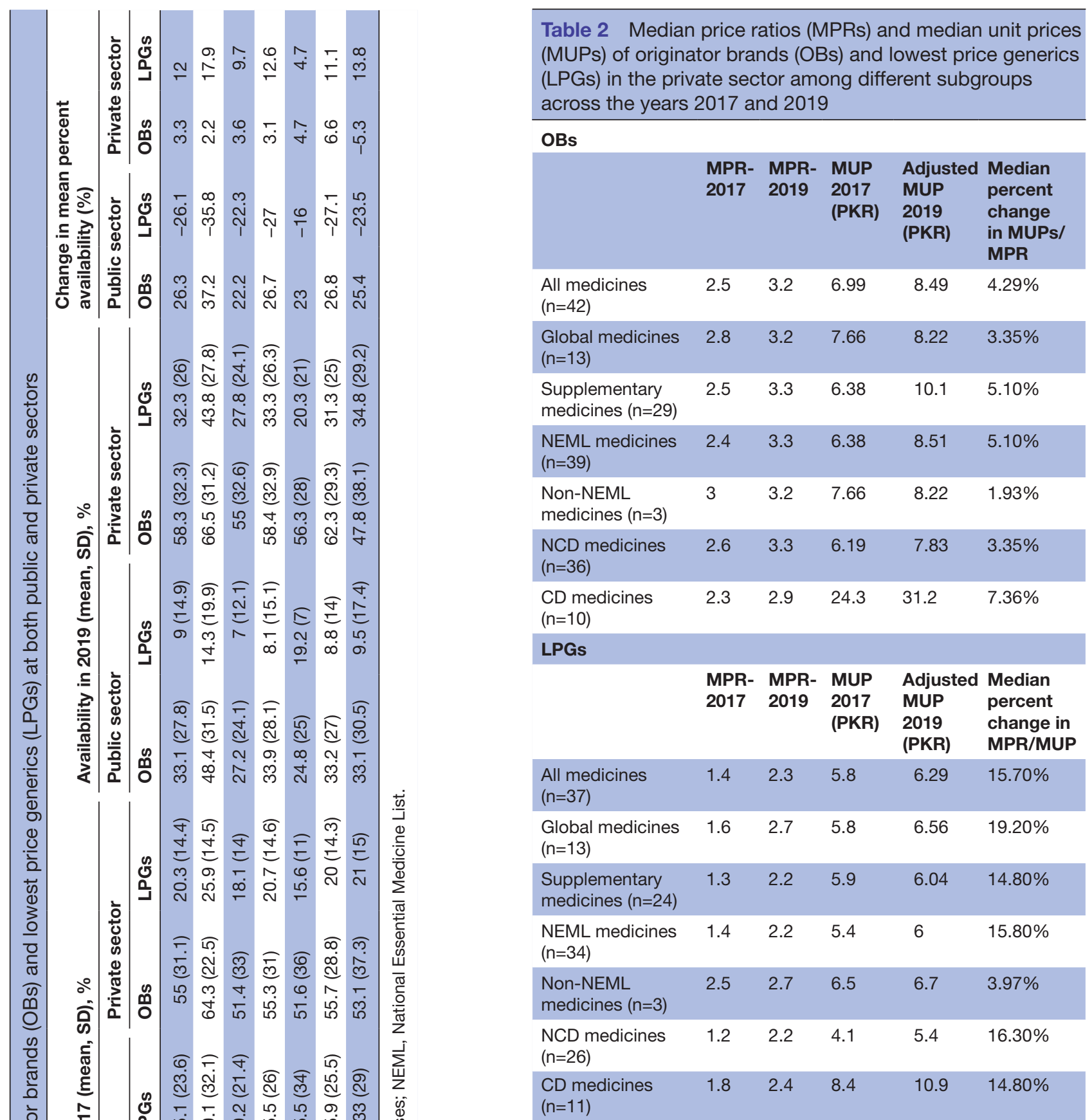

$\mathrm{CD}$, communicable diseases; NCD, non-communicable diseases; PKR, Pakistani rupee.

the adjusted MUPs and MPRs increased by a median of $15.7 \%(\mathrm{p}=0.002, \mathrm{p}=0.0002)$. In 2017, the MPRs of OBs ranged from 0.58 to 60.62 in 2017 and from 0.73 to 77.59 in 2019. Sixty-three per cent of the OBs had MPR of more than 2, whereas, in 2019, almost $75 \%$ of the OBs had MPR greater than 2.The MPRs of LPGs ranged from 0.42 to 19.95 in 2017 and from 0.39 to 19.89 in 2019. In 2017, for LPGs, the median value of MPR was less than 2 (ie, 1.36), while in 2019, this median value became greater than 2 (ie, 2.26). This means that many LPGs which were previously affordable got shifted to the high priced medicines category in 2019. The MUPs and MPRs for all OBs are 
given in online supplemental table S3 and those for all LPGs are given in online supplemental table S4.

The price data were also analysed in different subgroups, as shown in table 2. There was an increase in MUPs and MPRs for OBs of supplementary list of medicines compared with medicines from global list $(5.5 \%$ vs $3.35 \%)$. However, it was inverse in the case of LPGs, that is, the prices of global medicines increased compared with supplementary medicines $(19.2 \%$ vs $14.8 \%)$. Increment in the prices of NEML medicines was more as compared with non-NEML medicines. Next, we compared the medicines used for NCDs and IDs. Data suggested that the increase in the MUPs and MPRs of OBs for IDs was significant in comparison to NCDs (11.2\% vs $7.36 \%)$, whereas it was completely opposite in the case of LPGs, where the increase was greater for NCD than ID medicines $(16.3 \%$ vs $14.8 \%)$. It is also noteworthy that increase in prices for LPGs is more significant than OBs for all subgroups of medicines.

\section{Affordability}

Between 2017 and 2019, the median NDWs required for treatment with all OBs $(n=36)$ increased from 1.05 to 2 and from 0.5 to 0.7 for all LPGs $(\mathrm{n}=31)$, respectively. In 2019, the median percent increase in NDWs for LPGs $(\mathrm{n}=31)$ was much higher as compared with OBs $(\mathrm{n}=36)$, that is, $12.5 \% \quad(\mathrm{p}=0.008)$ and $3 \%(\mathrm{p}=0.081)$, respectively. So, an overall increase in NDWs for both OBs and LPGs was observed between 2017 and 2019. Similarly, the median treatment prices (MTPs) for OB and LPGs also increased significantly, that is, from 464 PKR to 563 PKR $(\mathrm{p}<0.001)$ and from 244 PKR to 350 PKR $(\mathrm{p}<0.001)$, respectively. The MTPs and NDWs for each medicine are given in online supplemental tables S5 and S6. The medicines were categorised into seven disease groups to further analyse changes in affordability between 2017 and 2019. In figure 2, a bar graph shows median NDWs required for both OBs and LPGs in each disease group, where the values above 1 were considered unaffordable. In 2017, the median NDWs of OBs to treat three types of diseases, that is, CVDs (1.2), diabetes (1.4) and ulcers

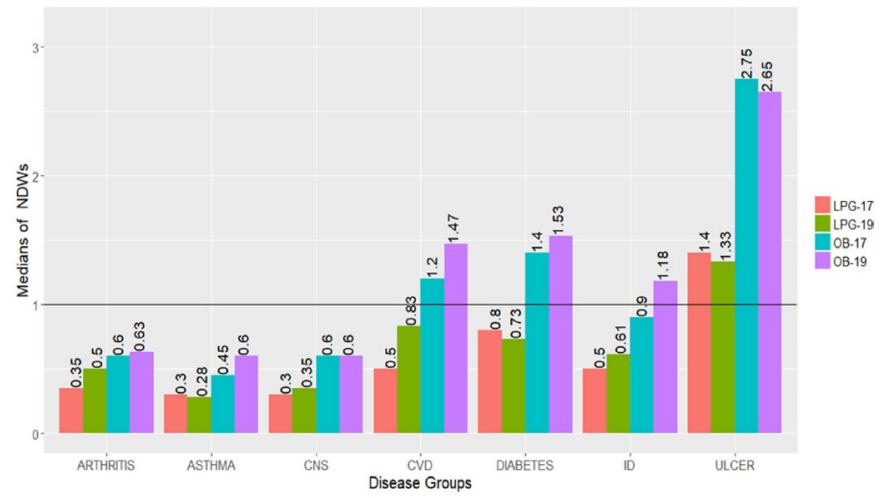

Figure 2 Bar graph of affordability of originator brands (OBs) and lowest price generics (LPGs) for different diseases in both years, that is, 2017 and 2019. CNS, central nervous system; CVD, cardiovascular disease; ID, infectious disease.
(2.75) was more than 1 , whereas in 2019 , medicines for another disease category made its place into this list, that is, the medicines for IDs (1.18). Compared with 2017, the median NDWs for all OBs increased in 2019, except for OBs acting on the central nervous system (CNS) and OBs to treat ulcers. The treatment for ulcer remained highly unaffordable in both years. The median NDWs for LPGs increased in 2019 for the treatment of arthritis, CNS disorders, CVDs and IDs, while the modest decrease in median NDWs for LPGs was observed for some diseases, that is, asthma, diabetes and ulcers, as shown in figure 2.

\section{DISCUSSION}

This study provides a valuable insight into the effects of NDPP 2018 associated changes on medicine prices, availability and affordability in both public and private healthcare sectors of Lahore division, Pakistan. The main objectives of this updated policy were to improve the access to EMs and to improvise rational drug pricing. Our study has shown that the overall availability of medicines improved in 2019 in comparison to 2017, that is, before the implementation of this policy, except for the LPGs in the public sector, demonstrating reduction. Overall, the medicine prices were increased significantly, making majority of the EMs used for the common ailments unaffordable, with a much higher price increases for LPGs in comparison to OBs. The medicines used to treat ulcers, diabetes and CVDs remained most unaffordable in both years. Despite the modest improvements in the availability of surveyed medicines after NDPP 2018, the increased unaffordability of the surveyed medicines earnestly requires significant revisions and improvements in the current pricing policy to ensure the affordability of surveyed medicines to the patients.

Despite improvements in the availability of medicines between 2017 and 2019, the availability of medicine remained below the optimal benchmark of $80 \% .{ }^{13}$ In the public sector, the availability of OBs improved remarkably probably be due to decentralisation procurement of medicines in the public sector. Before 2018, the medicines were procured centrally for all the public sector hospitals except for teaching hospitals, within a province. However, after 2018, the medicine procurement was decentralised for public sector hospital to allow hospitals in each district a free choice to select desired manufactures, thus, ending up in the selection of more OBs than LPGs, possibly due to quality concerns about medicines. Another factor that improved medicine availability after 2018 was authorisation of hospitals to acquire medicines directly without any delays. However, as practised in the previous central supply system, the medicines were received centrally from the manufacturers before reaching the concerned hospital with considerable effect on timely availability of medicines. In both years, the mean percentage availabilities for all medicines were found higher in the private sector than in the public sector, corroborating similar previous studies conducted in Bangladesh and Malawi 
in 2019. ${ }^{21} 22$ The overall availability of medicines from NEML was slightly better than non-NEML medicines in both public and private sectors. This might be attributed to the active role of DRAP in the revision and subsequent dissemination of the revised NEML, that is, NEML 2018. ${ }^{15}$ Furthermore, the public hospitals are encouraged to procure drugs from the latest NEML 2018 that has been standardised in line with the WHO EM model list 2017. ${ }^{23}$ Besides, a mobile application was launched in 2018 with user-friendly interface to better disseminate the information on enlisted medicines. ${ }^{24}$ So, the NDPP 2018 does not seem to be solely responsible for the availability of medicines.

Although we found some improvements in the availability of medicines, there was a substantial increase in medicine prices, making them inaccessible for most of the population. According to one estimate, approximately 46 million people are living below the national poverty line in Pakistan (as per 2015) ${ }^{8}$ The increases in prices of both OBs and LPGs may fairly be attributable to the NDPP 2018, which allows an annual increase in the prices of scheduled drugs up to $70 \%$ of CPI compared with $50 \%$ of CPI as per NDPP $2015 .^{9} 10$ These changes in price calculations seem to accentuate the substantial impact on overall prices of medicines, thus, making them more expensive. The increase in LPGs prices was more significant as compared with OBs, suggesting that with already expensive OBs, the price increase in LPGs would impoverish the overall access to medicines, imputable to the changes in the formula for LPGs (new entrants) price calculation. According to NDPP 2018, the MRP of new entrant first generic should be fixed at $20 \%$ less than that of OB compared with NDPP 2015, where it was $30 \%$ less than MRP of OB. Another possible variable is the prior availability of generics in the market for price calculation, where, according to NDPP 2018, the MRP of a new entrant (LPGs) was fixed equal to the highest MRP of the available generics in the market, while as per NDPP 2015 practice, MRP was fixed by taking the average of other generics in the market. Therefore, these changes in price calculating mechanisms might have led to higher prices of many new LPGs in the market. Hence, contrary to NDPP 2018's price steerage objectives, the increase in medicine prices was more distinct for NEML medicines as compared with non-NEML medicines.

Data from further analysis on affordability of standard treatment by selected OBs and LPGs suggested that majority of the medicines have become more unaffordable in 2019. When the affordability was compared for medicines of different disease groups, the three foremost unaffordable OBs were ones for treatment of ulcers, diabetes and CVDs. Additionally, the treatment for ulcers remained exceptionally unaffordable with OBs and LPGs in both years. Nevertheless, the treatment of CVDs and diabetes with LPGs remained affordable in 2017 but the NDWs for CVDs surpassed affordability threshold in 2019. Among the disease categories, NCDs harbour the top three unaffordable slots. It is noteworthy that the
Table 3 Maximum retail unit prices (MRPs) of originator brands (OBs) allowed by the government versus median unit prices (MUPs) found in private sector pharmacies

\begin{tabular}{llccc}
\hline Medicine name & $\begin{array}{l}\text { Strength } \\
\text { (dosage } \\
\text { form) }\end{array}$ & $\begin{array}{l}\text { Allowed } \\
\text { unit price } \\
\text { (PKR) }\end{array}$ & $\begin{array}{l}\text { Mup } \\
\text { 2019 } \\
\text { (PKR) }\end{array}$ & $\begin{array}{l}\text { Percentage } \\
\text { difference }\end{array}$ \\
\hline Aciclovir & $200 \mathrm{mg}$ (tab) & 52.6 & 75 & $42.5 \%$ \\
\hline Amlodipine & $5 \mathrm{mg}$ (tab) & 8.5 & 13 & $52.9 \%$ \\
\hline Amoxicillin & $250 \mathrm{mg}$ (cap) & 3.75 & 3.75 & $0 \%$ \\
\hline Amoxicillin & $500 \mathrm{mg}$ (cap) & 5.58 & 8.75 & $56.8 \%$ \\
\hline Atorvastatin & $20 \mathrm{mg}$ (cap) & 141.37 & 203.5 & $43.9 \%$ \\
\hline Bisoprolol & $5 \mathrm{mg}$ (tab) & 15.35 & 16.72 & $8.9 \%$ \\
\hline Carbamezipine & $200 \mathrm{mg}$ (tab) & 4 & 5 & $25 \%$ \\
\hline Ceftriaxone & $1 \mathrm{~g}$ (inj) & 783 & 783 & $0 \%$ \\
\hline Ciprofloxacin & $500 \mathrm{mg}$ (tab) & 39.25 & 52.5 & $33.7 \%$ \\
\hline Digoxin & $0.25 \mathrm{mg}$ (tab) & 1.75 & 2.68 & $53.1 \%$ \\
\hline Fluconazole & $200 \mathrm{mg}(\mathrm{cap})$ & 425 & 585 & $37.6 \%$ \\
\hline Insulin N & $100 \mathrm{IU}$ (vial) & 88.47 & 75.88 & $-14.2 \%$ \\
\hline Insulin R & $100 \mathrm{IU}$ (vial) & 93.88 & 75.88 & $-19.1 \%$ \\
\hline Methyldopa & $250 \mathrm{mg}$ (tab) & 7.71 & 8.1 & $5.05 \%$ \\
\hline Omeprazole & $20 \mathrm{mg}$ (cap) & 42.9 & 52.29 & $21.8 \%$ \\
\hline Propranolol & $40 \mathrm{mg}$ (tab) & 1.1 & 3.16 & $187.2 \%$ \\
\hline Pyremethamine+ & (25+500) mg & 12.01 & 12.02 & $0.08 \%$ \\
\hline Sulfadoxime & (tab) & & & \\
\hline Simvastatin & $20 \mathrm{mg}$ (cap) & 47.01 & 68 & $44.6 \%$ \\
\hline Medians & & 27.3 & 34.505 & $29.3 \%$ \\
\hline & & & & \\
\hline
\end{tabular}

burden of NCDs is increasing worldwide and is responsible for higher mortality rates than all other diseases combined. $^{25-27}$ The CVDs, diabetes, cancer and chronic respiratory diseases are responsible for about $80 \%$ of these deaths. ${ }^{28}$ Pakistan is among top 10 countries where prevalence of diabetes is very high. Besides, one-third of Pakistanis, above 45 years of age, have hypertension. ${ }^{29-31}$ Thus, the unaffordability of the EMs for NCDs, such as CVDs and diabetes, has worse bearing on affordabilityassociated therapeutic outcomes that ultimately leads to increased morbidity and mortality due to uncontrolled disease.

Additionally, we also compared the median drug prices in 2019 with the prices published/allowed by DRAP in its latest Statutory Regulatory Orders (SROs). On 31 December 2018, the DRAP revised and published the maximum retail prices (MRPs) of about 1084 drugs through SRO-1608, SRO-1609 and SRO-1610. ${ }^{32}$ Eighteen OBs from our study sample were part of these price revisions (table 3). Surprisingly, after comparing the MUPs of 18 selected OBs with prices allowed by the government, we found that most of the OBs, 14 out of 18 , were sold at higher prices than the allowable prices-with median percent increase of $29.37 \%$. These data suggested that these intentional malpractices by the drug sellers might be driven by poor price control regulation by price enforcement authorities. Therefore, the current drug pricing policy NDPP 2018 is not the sole reason for the 
price hike. Most probably main stakeholders in the drug supply chain are also contributing towards medicine inflated prices. Thus, it is reasonable to deduce that these factors may interfere with measuring the direct impact of current pricing policy.

Although the formation of a national scale pricing policy is laudable but it seems to be a collection of drug price calculation formulas only. It should also include the mechanism for price monitoring, an aspect that seems to be one of the major reasons behind failure to achieve the goals of NDPP 2018. Inclusion of the WHO/HAI-based surveys on a regular basis could also be an option, in this case. The WHO has developed a mobile application named 'WHO Essential Medicines and Health Products Price and Availability Monitoring (WHO EMP MedMon)', which can be used to collect and analyse price and availability data from healthcare outlets. This application is based on standard WHO/HAI methodology and it can be used both online and offline, making it both time saving and cost-effective. ${ }^{33}$ The DIs or a third party can be given this responsibility to monitor and report the prices using WHO EMP MedMon on a regular basis, ensuring the compliance by drug manufacturers and sellers to NDPP. There could be many other policy implications having an impact on drug pricing, availability and affordability. The procurement of medicines should be strictly based on the NEML. Clear-cut mechanism for NEMLbased procurement should be devised and implemented especially in the public sector hospitals. Besides, the hospital pharmacy and therapeutics committees must actively evaluate the safety, efficacy and cost-effectiveness of drugs before purchasing. Pharmaco-economic evaluations of drugs must be promoted by allocating research funds to experts. Not all drugs should be fully reimbursed in the hospitals, only EMs must be included in this list. Hence, the profits from other drugs can be used to purchase essential drugs when needed. Smooth functioning of the drug supply chain with proper quality control must be ensured. The current inflated prices would have a grave impact on the access to EMs, especially for the low-income and middle-income population of Pakistan. Thus, there is dire need to develop clearer evidencebased and stringent price control policy, especially for EMs. Exempting or reducing taxes and tariffs on EMs and promoting local generic manufacture by providing subsidies on raw materials may improve both the availability and the affordability of these medicines. While using the ERP mechanism, the reference countries should be chosen critically, for example, countries with similar pharmaceutical market and economic status. For costly medicines, regressive markups must be encouraged over progressive markups. The drug prices must be monitored on a regular basis using a validated and well-designed scientific methodology, and pricing policy must be revised based on such evidence. The EMs for most prevalent diseases such as diabetes and CVDs must be preferentially made affordable by devising some specific pricing strategies for these medicines. Besides, efforts must be made to enforce the pricing policy effectively by introducing reward and punishment system to induce a healthy competition among the drug manufacturers and sellers.
Although this study provides an objective evidence to the policy makers for improving the current pricing policies, it has some limitations as well. The study includes medicines with specific strengths and dosage forms to compare with IRPs. There might be other strengths/dosage forms of the surveyed medicines, available in the health facilities, so the availability of the medicines may be underestimated. Affordability was calculated for single medicine for each disease, whereas patients are usually taking more than one drug at a time, underestimating the extent of affordability of a specific treatment for a specific disease. Moreover, the post survey was conducted after about a year from the launch of new drug pricing policy 2018, so the results do not reflect the long-term impact of the policy. Further surveys could be conducted in the future to gauge the long-term effects of the policy, as it was done by Fang et al in two such surveys conducted after the health reform in China. ${ }^{17}$

In conclusion, the availability of medicines has been improved after the launch of a new drug pricing policy by Pakistani government but it is still below the benchmark, thus, forcing the patients to buy medicines from the private sector at their own expense. The prices of both LPGs and OBs of EMs have increased remarkably in 2019 compared with 2017. The medicines to treat most prevalent NCD (diabetes and CVDs) have become more expensive and unaffordable. The maximum retail prices of several OBs have been illegally increased in the market, adding more burden on patients' pockets. Thus, the pricing policy should be improved with strict price control measures, especially for the EMs, such as ensuring transparency on the costs of drug development process and distribution, NEML-based procurement, and reduction in the taxes and tariffs on local production of EMs.

\section{Author affiliations}

${ }^{1}$ Department of Pharmacy Administration and Clinical Pharmacy, School of Pharmacy, Xi'an Jiaotong University, Shaanxi, China

${ }^{2}$ Center for Drug Safety and Policy Research, Xian Jiaotong University, Shaanxi, China

${ }^{3}$ Shaanxi Centre for Health Reform and Development Research, Shaanxi, China ${ }^{4}$ Department of Pharmaceutics, University of the Punjab, Lahore, Pakistan ${ }^{5}$ Faculty of Pharmacy, The University of Lahore, Lahore, Pakistan ${ }^{6}$ Department of Pharmacy, School of Applied Sciences, University of Huddersfield, Huddersfield, West Yorkshire, UK

Acknowledgements We are thankful to Dr Margaret Ewen, senior projects manager, medicine prices at Health Action International (HAl), for helping us in designing the study. We are also grateful to Dr Hira Azhar for editing this manuscript. We would like to acknowledge the pharmacists from the surveyed institutions, data collectors and partners from research area who made this research possible.

Contributors AS, ZUDB and YF conceptualised the study. ZS, HS, AS and ZUDB designed the methodology. ZS, HS and AS trained the data collectors and obtained the data. YF provided resources and supervised the project. ZS worked as survey area manager. MMA, AHG, NA, FUK and MZ did data cleaning, validation and entry. AS, $\mathrm{HS}, \mathrm{CY}$ and $\mathrm{MJ}$ analysed the data. AS and HS wrote the original draft. YF, ZUDB, $\mathrm{ZS}, \mathrm{CY}, \mathrm{MJ}$ and $\mathrm{WJ}$ reviewed and edited the manuscript.

Funding This work was funded by the 'Young Talent Support Plan', 'High Achiever Plan' of Health Science Center, Xi'an Jiaotong University and the Central University Basic Research Fund (2015qngz05).

Competing interests None declared. 
Patient and public involvement Patients and/or the public were not involved in the design, or conduct, or reporting or dissemination plans of this research.

Patient consent for publication Not required.

Ethics approval Ethics approval was obtained from the Medical Ethics Committee of Xi'an Jiaotong University under study ID 2019-067.

Provenance and peer review Not commissioned; externally peer reviewed.

Data availability statement Data are available in a public, open access repository. Extra data can be accessed via the Dryad data repository at http://datadryad.org/ with the doi:10.5061/dryad.tqjq2bvwq.

Open access This is an open access article distributed in accordance with the Creative Commons Attribution Non Commercial (CC BY-NC 4.0) license, which permits others to distribute, remix, adapt, build upon this work non-commercially, and license their derivative works on different terms, provided the original work is properly cited, appropriate credit is given, any changes made indicated, and the use is non-commercial. See: http://creativecommons.org/licenses/by-nc/4.0/.

\section{ORCID iDs}

Amna Saeed http://orcid.org/0000-0002-0284-0260

Hamid Saeed http://orcid.org/0000-0002-1400-4825

Faiz Ullah Khan http://orcid.org/0000-0002-1022-8688

\section{REFERENCES}

1 United Nations Development Group, United Nations. Statistical division. indicators for monitoring the millennium development goals: definitions, rationale, concepts and sources. 95. United Nations Publications, 2003.

2 World Health Organization. Medicine prices and access to medicines in the eastern Mediterranean region, 2007.

3 Lee KS, Shahidullah A, Zaidi STR, et al. The crux of the medicine prices' controversy in Pakistan. Front Pharmacol 2017;8:504.

4 Kiani A, Qadeer A, Mirza Z, et al. Prices, availability and affordability of medicines in Pakistan. Geneva: Health Action International, 2006.

5 Zaidi S, Bigdeli M, Aleem N, et al. Access to essential medicines in Pakistan: policy and health systems research concerns. PLoS One 2013;8:e63515.

6 Cameron A, Ewen M, Ross-Degnan D, et al. Medicine prices, availability, and affordability in 36 developing and middle-income countries: a secondary analysis. Lancet 2009;373:240-9.

7 Saeed A, Saeed H, Saleem Z, et al. Evaluation of prices, availability and affordability of essential medicines in Lahore division, Pakistan: a cross-sectional survey using WHO/HAI methodology. PLoS One 2019;14:e0216122.

8 The World Bank. Poverty and equity data portal, 2015. Available: $\mathrm{http}: / /$ povertydata.worldbank.org/poverty/country/PAK [Accessed 16 Jun 2019].

9 Costing and Pricing Division, Drug Regulatory Authority of Pakistan. Drug pricing policy, 2015.

10 Costing and Pricing Division, Drug Regulatory Authority of Pakistan. Drug pricing policy, 2018.

11 Durrani F. Increase in drug prices highest in last 40 years, 2019. Available: https://www.thenews.com.pk/print/454864-increase-indrug-prices-highest-in-last-40-years [Accessed 3 Sep 2019].

12 Junaidi I. Minister orders crackdown on firms increasing medicine prices. DAWN News, 2019. Available: https://www.dawn.com/news/ 1473573 [Accessed 3 Sep 2019].
13 World Health Organization. Measuring medicine prices, availability, affordability and price components, 2008.

14 Pakistan Bureau of Statistics. Population census, 2017. Available: http://www.pbs.gov.pk/content/population-census [Accessed 18 Aug 2019].

15 Drug Regulatory Authority of Pakistan. National essential medicines list, 2018.

16 Yang H, Dib HH, Zhu M, et al. Prices, availability and affordability of essential medicines in rural areas of Hubei Province, China. Health Policy Plan 2010;25:219-29.

17 Fang Y, Wagner AK, Yang S, et al. Access to affordable medicines after health reform: evidence from two cross-sectional surveys in Shaanxi Province, Western China. Lancet Glob Health 2013;1:e227-37.

18 Management Sciences for Health. International medical products price guide, 2015.

19 Pakistan Bureau of Statistics, Government of Pakistan. Price statistics. Available: http://www.pbs.gov.pk/content/officiallypublished-consumer-prices-data-imf-template [Accessed 18 Aug 2019].

20 Wage Indicator Foundation. Minimum wages in Pakistan, 2018. Available: https://wageindicator.org/Wageindicatorfoundation [Accessed 15 Apr 2019].

21 Kasonde L, Tordrup D, Naheed A, et al. Evaluating medicine prices, availability and affordability in Bangladesh using World health organisation and health action international methodology. BMC Health Serv Res 2019;19:383.

22 Khuluza F, Haefele-Abah C. The availability, prices and affordability of essential medicines in Malawi: a cross-sectional study. PLoS One 2019;14:e0212125.

23 Jabri P. National essential medicine list -2018 to be implemented in all provinces: Kiani. business recorder, 2018. Available: https://www. brecorder.com/2018/10/06/444080/national-essential-medicine-list2018-to-be-implemented-in-all-provinces-kiani/ [Accessed 3 Sep 2019].

24 Pakistan Today. Govt. launches WHO backed national essential medicine list, 2018. Available: https://www.pakistantoday.com.pk/ 2018/10/05/govt-launches-who-backed-national-essential-medicinelist/ [Accessed 3 Sep 2019].

25 Dans A, Ng N, Varghese C, et al. The rise of chronic noncommunicable diseases in Southeast Asia: time for action. Lancet 2011;377:680-9.

26 Siegel KR, Patel SA, Ali MK. Non-communicable diseases in South Asia: contemporary perspectives. Br Med Bull 2014;111:31-44.

27 Habib SH, Saha S. Burden of non-communicable disease: global overview. Diabetes Metab Syndr 2010;4:41-7.

28 World Health Organization. Global action plan for the prevention and control of non-communicable diseases 2013-2020, 2013.

29 Ghaffar A, Reddy KS, Singhi M. Burden of non-communicable diseases in South Asia. BMJ 2004;328:807-10.

30 Jafar TH, Haaland BA, Rahman A, et al. Non-communicable diseases and injuries in Pakistan: strategic priorities. Lancet 2013;381:2281-90.

31 Wasay M, Zaidi S, Khan M, et al. Non communicable diseases in Pakistan: burden, challenges and way forward for health care authorities. J Pak Med Assoc 2014;64:1218.

32 Drug Regulatory Authority of Pakistan. SRO: pricing division 2018. Available: https://www.dra.gov.pk/Home/Pricing [Accessed 17 Jul 2019].

33 World Health Organization. MedMon - WHO essential medicines and health products price and availability monitoring mobile application, 2016. Available: https://www.who.int/medicines/areas/policy/ monitoring/empmedmon/en/ [Accessed 30 Mar 2020]. 